\section{Improved Techniques For Imaging \\ Of Three-Dimensional Transparent Specimens In Advanced Darkfield And Interference Contrast Modes}

\author{
Jörg Piper \\ Clinic Meduna \\ Bad Bertrich, Germany \\ JPu.MP@t-online.de
}

\section{Introduction}

In light microscopy, dark field and interference contrast are widely used for examination of transparent specimens. These methods both suffer from various limitations when photomicrographs have to be taken from fine details, especially in three-dimensional specimens requiring a large depth of field.

In common dark field illumination, the condenser either is not equipped with an aperture diaphragm, or an existing condenser diaphragm has to remain in the wide-open position. Thus, the depth of field is lower than in bright field images. Moreover, dark field imaging is associated with marginal blooming, especially in linear structures exhibiting with large differences in phase or density (e.g. cell walls, edges in crystals and other mineralogical material). As specimens are illuminated by oblique light that comes from the periphery of the illuminating apparatus, fine structures inside them may only be barely or sometimes not visible. Last, dark field images are characterized by a high dynamic range, i.e. strong differences in brightness, so that some parts of the specimen can be over- or underexposed in photomicrographs.

Similarly, interference contrast is characterized by a small depth of field. When the vertical resolution of the optical system is lower than the local thickness of the specimen, most structures are out of focus and are not visible. In this case, specimens appear like optical sections when interference contrast is carried out so that their three-dimensional architecture can often not be clearly presented in typical single shot images.

In this article, several modifications of existing optical equipment have been made in order to achieve fundamental improvements of the global image quality in darkfield imaging especially in observations of three-dimensional specimens. Moreover, some software-based solutions described are suitable for further optimization in images from three-dimensional specimens taken in interference contrast or darkfield illumination.

\section{Materials and methods}

An industrial microscope (Leitz/Leica SM Lux HL), constructed for wafer inspections based on infinite optics and designed for various
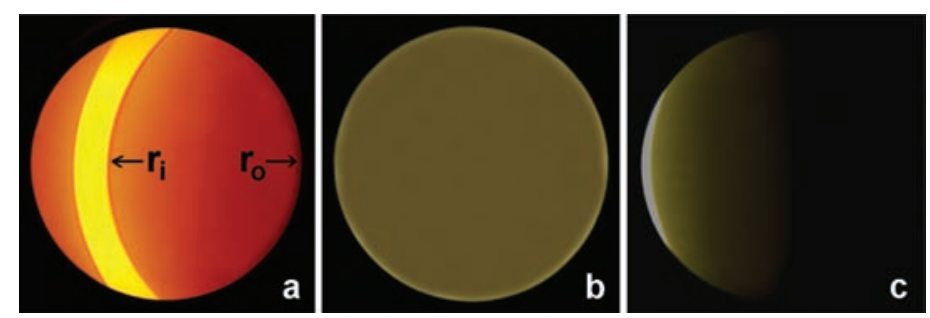

Fig. 1: Achievement of „condenser aperture reduction darkfield“ by use of a phase contrast condenser, controlled by a phase telescope

$r_{i}=$ interior radius of the light ring (condenser annulus),

$r_{0}=$ interior radius of the object field or optical circumference of the objective

a: off-centered position of the light ring

b: alignment for concentric condenser aperture reduction darkfield $\left(360^{\circ}\right.$ illumination)

$c$ : discrete off-centered position for eccentric condenser aperture reduction darkfield (oblique illumination) illumination techniques in epiillumination and transmitted light, was used for several optical experiments resulting in the development of concentric and eccentric "condenser aperture reduction dark field". Because of the special design of this microscope, "condenser aperture reduction darkfield" could also be combined with dark field in epi-illumination (so-called "superimposed darkfield"). Optical tests were carried out with special objectives for darkfield examination in epi-illumination and special lenses designed with an integrated iris diaphragm for improved darkfield illumination in transmitted light.

Based on mirror lenses, "axial darkfield" was also evaluated as a further attractive variant of darkfield illumination. These experiments were carried out with common laboratory microscopes based on 160 or $170 \mathrm{~mm}$ tube lengths equipped with several mirror objectives in different optical constructions (COOKE and CASSEGRAIN-SCHWARZSCHILD types). In detail, the following mirror lenses were used:

- Catadioptric CASSEGRAINSCHWARZSCHILD lenses made by Carl Zeiss Jena (CZJ), designed for examinations of coverslip preparations: $16 \times(\mathrm{NA}$ $0.32), 50 \times(\mathrm{NA}(0.65)$ and $100 \times$ (NA 0.65) dry systems, 110x (NA 0.90) and $125 \times$ (NA 0.90) water immersions

- 40× (NA 0.65) catoptric CASSEGRAIN-SCHWARZSCHILD lens (dry system) from CZJ, designed for examinations of specimens without coverslip

- 40× (NA 0.57) catadioptric

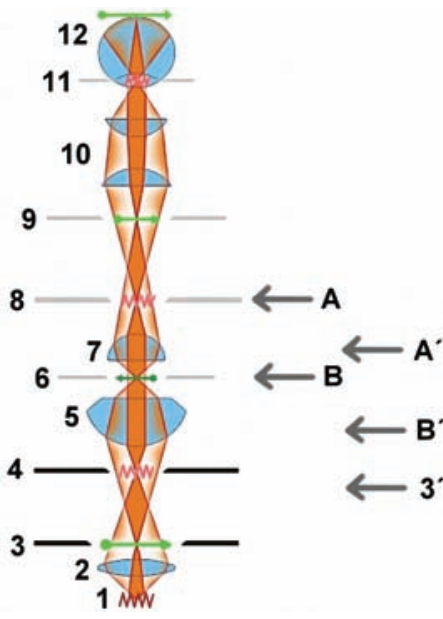

Fig. 2: Pathways of the illuminating and imaging light in a conventional microscope, shift of projection planes in condenser aperture reduction darkfield (modified from Linkenheld)

$$
\begin{aligned}
& \text { 1. }=\text { light source } \\
& \text { 2. = collector } \\
& \text { 3. = field diaphragm (for }
\end{aligned}
$$

KÖHLER illumination)

4. = condenser aperture diaphragm

5. = condenser

6. $=$ specimen (green arrow)

7. $=$ objective

8. = backfocal plane (exit pupil) of the objective

9. = intermediate image

10. $=$ eyepiece

11. = backfocal plane (exit pupil) of the ocular

12. $=$ eye

$A=$ projection field of the condenser aperture diaphragm in conventional illumination

$A^{\prime}=$ shift of plane $A$ in condenser aperture reduction darkfield

$B=$ projection plane of the field diaphragm in conventional illumination

$B^{\prime}=$ shift of plane $B$ in condenser aperture reduction darkfield

$3^{\prime}=$ position of an effectively working field diaphragm in condenser aperture reduction darkfield

COOKE lens (real magnification: $50 \times$ ), designed as a long distance system (working distance: $15 \mathrm{~mm}$ !) suitable for examinations of specimens prepared in each mode (uncovered or covered by a coverslip).

Using the same laboratory microscopes, interference contrast was carried out in a conventional manner. Software-based, the resulting images were transformed into a "digitized interference contrast" file.

For computer-based postprocessing, the following software solutions were used: Combine Z 5 [4], Picolay [1] and Helicon Focus [5] for enhancements of the depth of field, and Photomatix Pro [3] for high dynamic range rendering (HDR).

All techniques were tested on some shells of radiolarians and diatoms arranged in cover slip preparations. Complementary evaluations were made on colorless crystallizations prepared without coverslip and 


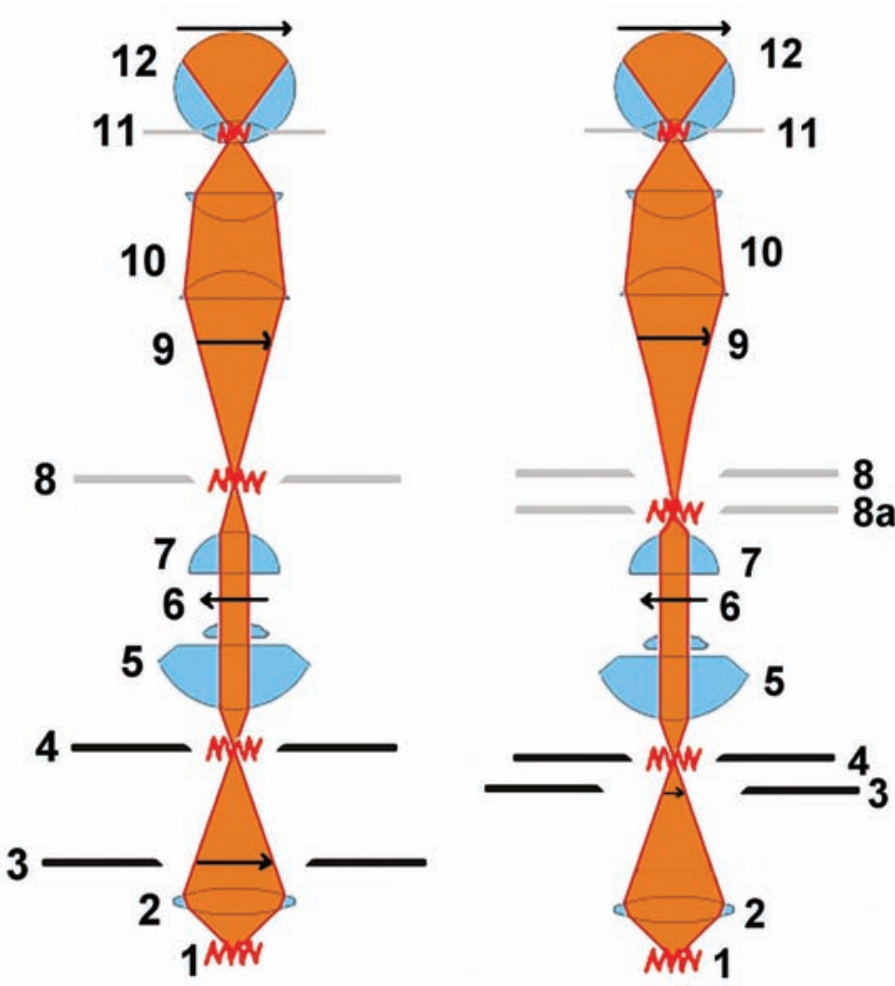

Fig. 3: Pathway of the illuminating light in conventional mode (a) and in a modified equipment designed for condenser aperture reduction darkfield $(b)$. The projection plane of the aperture diaphragm is shifted from plane 8 into plane 8a. Other labels as shown in fig. 2 (modified from Linkenheld).

on additional biological specimens. The photomicrographs were taken with an Olympus Camedia C-7070 digital camera equipped with Leitz/ Leica Vario Photo Oculars $5 \times-12.5 \times$ or $6.5-12.5 \times$.

\section{Implementation of "condenser aperture reduction darkfield"}

To obtain "condenser aperture reduction darkfield", the normally used condenser of the SM Lux HL-microscope constructed for infinite lenses was replaced by an universal condenser for phase contrast examinations originally designed for microscopes with a $170 \mathrm{~mm}$ tube length (Leitz/Leica ZERNIKE condenser 402a). The focal intercept in this ZERNIKE condenser (NA 0.9) was $11 \mathrm{~mm}$ lower than in the other condenser (NA 0.6) normally used with the SM Lux HL-microscope as recommended by the manufacturer. The ZERNIKE condenser was fitted with several annular-shaped transparent masks in different diameters, as used in phase contrast illumination. In one of these light rings (fig. 1a), the inner diameter $\left(r_{i}\right)$ was just a little greater than the diameter of the object field or the optical diameter of the respective objective $\left(r_{0}\right)$. When the light ring was adjusted in a centered position (fig. 1b), a black background could be achieved, and the specimen illuminated

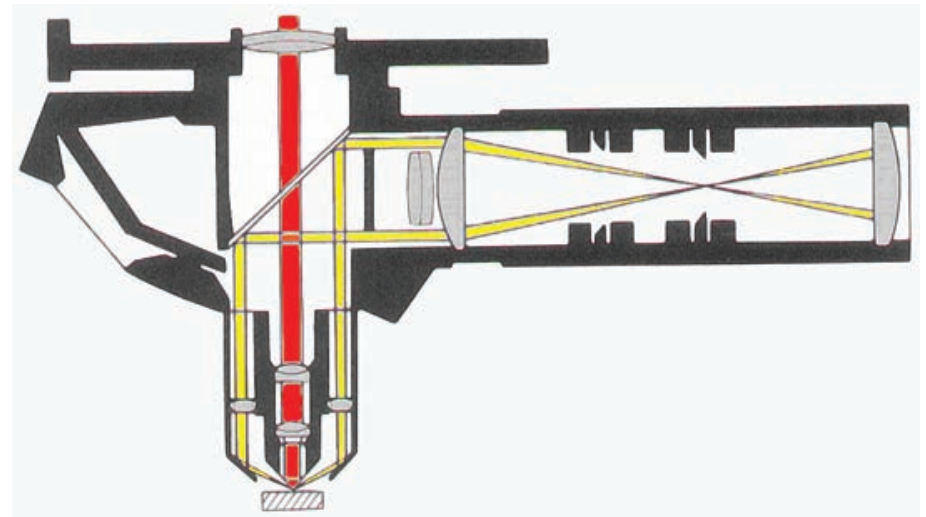

Fig. 4: Optical pathway in darkfield based on epi-illumination in a concentric circular manner $\left(360^{\circ}\right)$. When the light ring was moved to an off-centered position by a tiny shift (fig. 1c), oblique illumination effects could be achieved so that the three-dimensional relief of the specimen was accentuated while the background was moderately brightened. Summing up, two illumination modes could be achieved in this way: concentric and eccentric condenser aperture reduction darkfield.

The condenser replacement described above lead to fundamental modifications of the illuminating light path (fig. 2 and 3). While fig. 2 shows the pathways of both relevant light components - the illuminating and the imaging light beams-, fig. 3 demonstrates the basic modifications selectively for the illuminating light path.

As shown in fig. 2, in normal optical configurations, the condenser iris diaphragm (4) is projected into the back focal plane (exit pupil) of the objective (8, plane A), and the field diaphragm (3), necessary for KÖHLER illumination, is projected into the object plane / object field (6, plane $\mathrm{B})$. When the standard condenser constructed for infinite lenses is replaced by a condenser designed for a finite tube length, the positions of the projection planes A and B are both shifted into the corresponding planes A' and B'. Thus, the original field diaphragm no longer acts as a KÖHLER diaphragm, and the original condenser diaphragm is projected into a medium position (plane $8 \mathrm{a}$ in fig. $3 \mathbf{b}$ ) situated between the specimen plane (6) and the objective's back focal plane (8). According to our experiments, in this abnormal configuration, a separate iris diaphragm can be situated near the condenser diaphragm (see arrow 3' in fig. 2 and plane 3 in fig. $\mathbf{3 b}$ ) so that it is projected onto the specimen plane and can act as a field diaphragm in the normal manner.
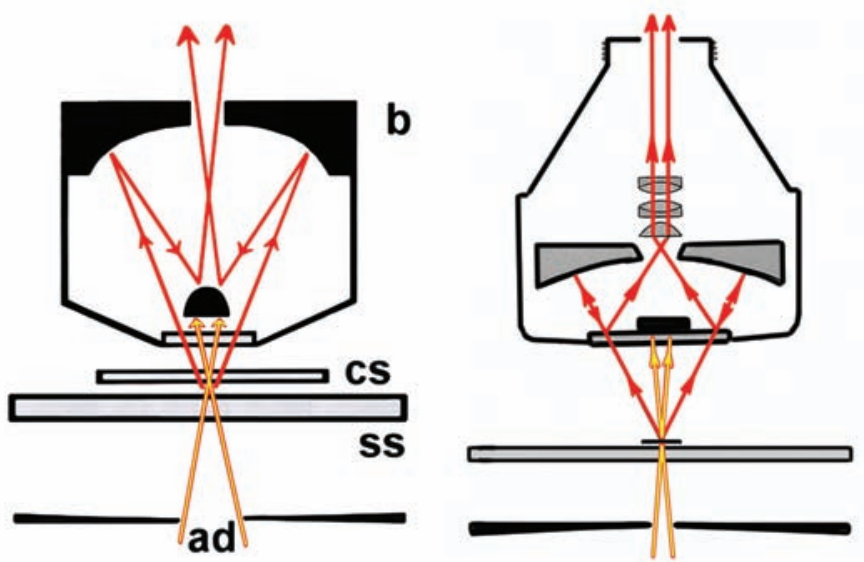

Fig. 5: (left) Axial darkfield (luminance contrast), light path in a CASSEGRAIN-SCHWARZSCHILD mirror objective. ad = condenser aperture diaphragm ss = specimen slides, $c s=$ cover slip

Fig. 6: (right) Axial darkfield (luminance contrast), light path in a COOKE mirror objective.)

Because of this modification in the optical design, the condenser iris diaphragm works in a similar way as the standard field diaphragm. The illuminating light running through the light ring within the condenser is no longer stopped when the condenser aperture diaphragm is closed. Therefore, the visible depth of field can be significantly enhanced by closing the condenser diaphragm. When the objective is fitted with an additional integrated iris diaphragm mounted in the back focal plane, the final depth of field can be enhanced even more as the projection plane of the condenser diaphragm is different from the plane of the diaphragm within the objective.

The illuminating light path is also modified with regard to the angle of incidence, because the focal intercept of the ZERNIKE condenser is 11 $\mathrm{mm}$ lower than it would be using the original condenser. In conventional darkfield, the illuminating light beams travel to the specimen from the periphery so that the specimen is illuminated in an oblique manner. In 


\section{DIATOME} diamond knives

\section{Development, Manufacturing, and Customer Service since 1970}

What have we achieved in this period?

ultra $45^{\circ}$ the first diamond knife with an absolutely score-free, hydrophilic cutting edge.

semi the first diamond knife for alternating sectioning ultrathin/semithin.

cryo the diamond knife for sectioning at low temperature.

histo the first diamond knife for semithin sections for light microscopy.

ultra $35^{\circ}$ the diamond knife for optimized sectioning results in almost all applications.

STATIC LINE II the ionizer for eliminating electrostatic charging in ultramicrotomy.

cryo-P a cryo knife with a patented platform for section pick up.

cryo immuno the optimized cryo diamond knife for the Tokuyasu technique.

ultra sonic the oscillating diamond knife for room temperature sectioning.

cryotrim 45 and 25 optimizing trimming with diamond blades.

ultra AFM \& cryo AFM the first diamond knives for AFM at room and low temperatures.

cryo $25^{\circ}$ for sectioning frozen hydrated specimens.

What services can we offer you?

Technical assistance in all fields of ultramicrotomy.

- Free sectioning tests for all types of samples.

I Make use of our many years of experience in perfecting our knives.

Custom knives, tools, and boats.

I Special purchase programs.

- Workshops and training.

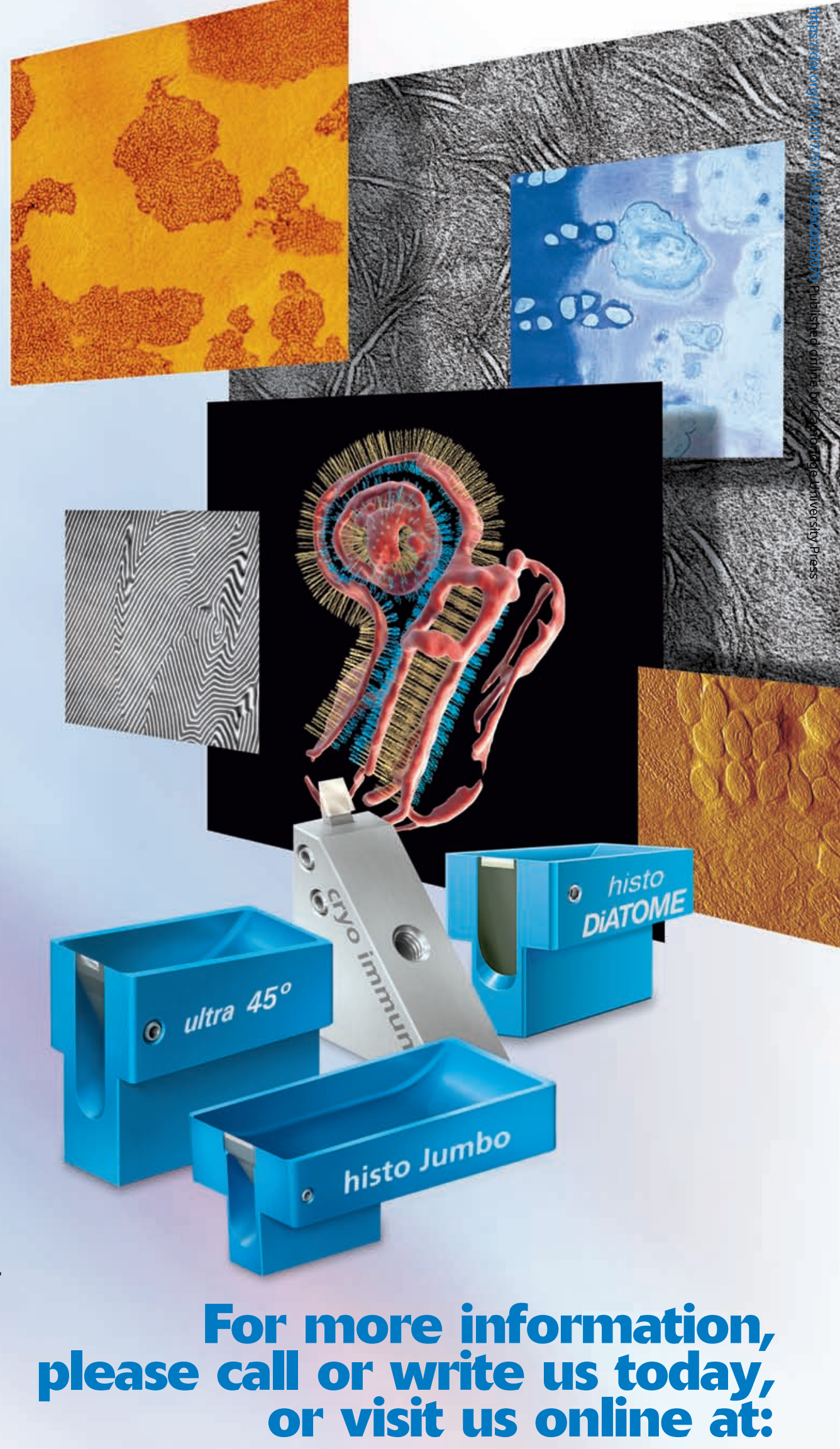

www.emsdiasum.com

DIATOME

for all your sectioning requirements
P.O. Box $410 \bullet 1560$ Industry Rd. Hatfield, Pa 19440

(215) 412-8390 • Toll Free: 1-(800) 523-5874

Fax: (215) 412-8450 or 8452

email:sgkcck@aol.com•stacie@ems-secure.com 


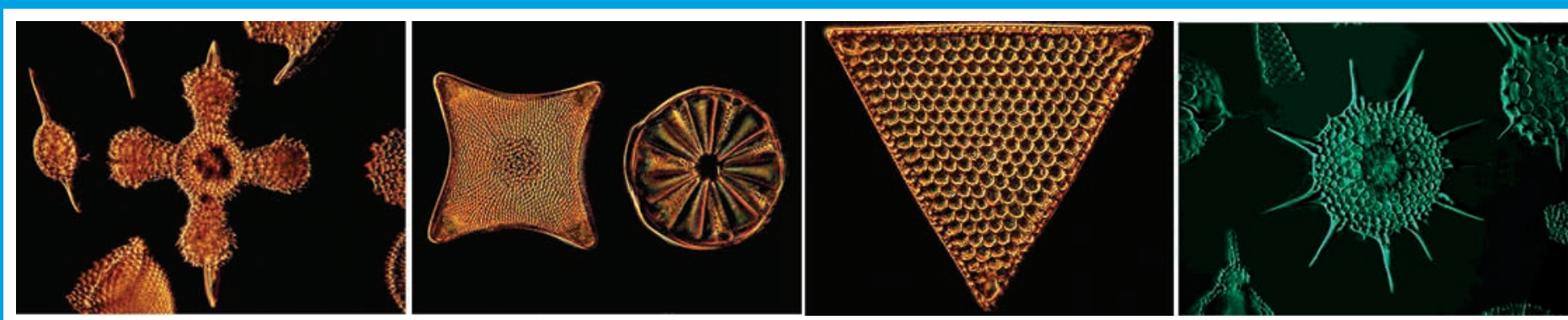

Left to right -Fig. 7: Radiolarians, objective 20x, concentric aperture reduction darkfield, horizontal field width (HFW): $0.60 \mathrm{~mm}$

Fig. 8: Diatoms, objective 20x, concentric aperture reduction darkfield, HFW: $0.25 \mathrm{~mm}$

Fig. 9: Diatom, objective 20×, concentric aperture reduction darkfield, HFW: $0.10 \mathrm{~mm}$

Fig. 10: Radiolarians, objective $20 \times$, eccentric aperture reduction darkfield, monochromatic green light $(\mathrm{O}-\mathrm{III}$ filter, $\lambda=500 \mathrm{~nm})$, discrete brightening of the background, HFW: $0.20 \mathrm{~mm}$
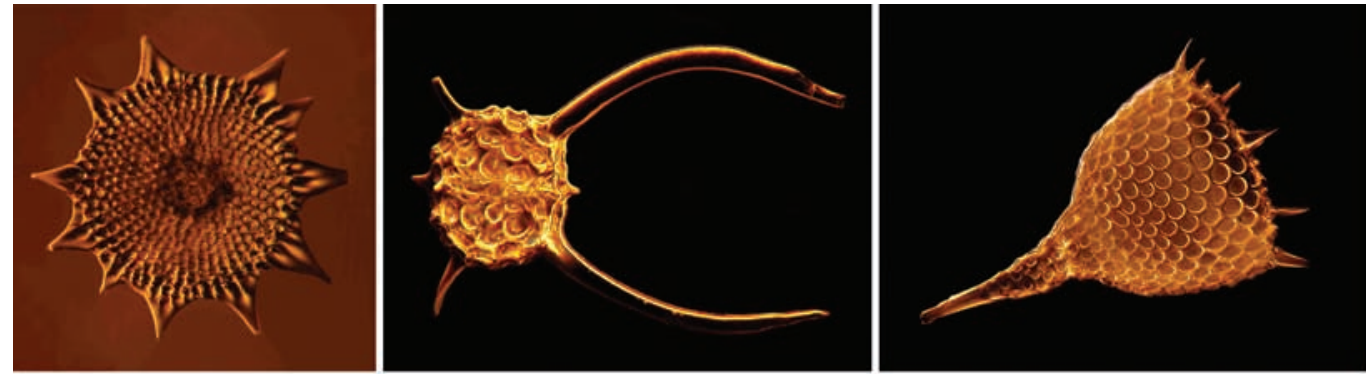

Left to right - Fig. 11: Radiolarian, objective 20×, eccentric aperture reduction darkfield, halogen bulb light, moderate brightening of the background, HFW: $0.27 \mathrm{~mm}$

Fig. 12: Radiolarian, objective iris $30 \times$, concentric aperture reduction darkfield, HFW: $0.25 \mathrm{~mm}$

Fig. 13: Radiolarian, objective iris $30 \times$, moderate eccentric aperture reduction darkfield, HFW: $0.25 \mathrm{~mm}$

Fig. 14: Radiolarians, objective 20×, darkfield in epi-illumination. HFW: $0.35 \mathrm{~mm}$

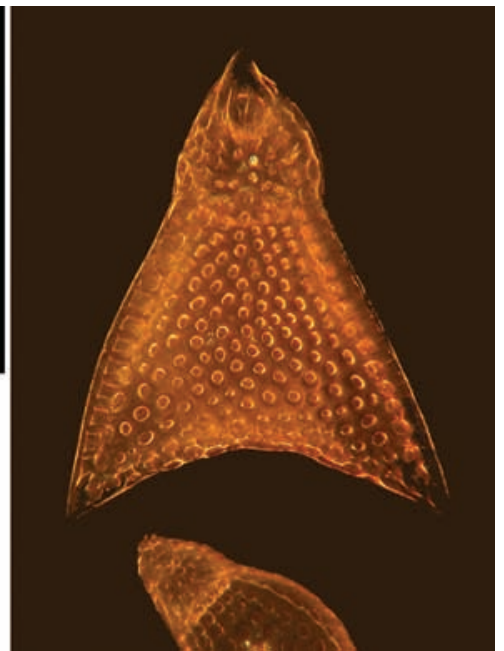

"condenser aperture reduction darkfield," the illuminating light travels at a higher angle so that the specimen is more centrically illuminated.

\section{Implementation of "superimposed darkfield"}

When a suitable illuminator for epi-illumination is available (fig. 4), "condenser aperture reduction darkfield" that is carried out in transmitted light, can be combined with darkfield in epi-illumination. In this case, the visual information can be accentuated still more, when both components of the illuminating light are filtered in different colors. By use of monochromatic narrow band filters, the resolution and sharpness of the resulting image can be optimized more - up to the physical limit of the respective optical system. In some specimens, fine superficial structures can be impressively documented in darkfield when epi-illumination is carried out.

\section{Implementation of "axial dark field"}

By use of mirror objectives, excellent axial dark field illumination can be achieved with a common condenser for bright field illumination. For this purpose, the condenser has to be centered most exactly, and the condenser iris diaphragm has to be turned to its maximum closed position. The field diaphragm has also to be closed adequately. In this arrangement, the specimen is illuminated in an axial direction by a very small central light beam congruent with the optical axis. Within a CASSEGRAIN-SCHWARZSCHILD mirror lens, all illuminating light components are completely blocked by the non transparent back side of the centric convex mirror that acts as a beam stop in this special case (fig. 5). When a COOKE mirror system is used, the illuminating light is blocked in the same way by a small centric light stop situated in the middle of the semi transparent plane-parallel front mirror (fig. 6). As the small illuminating central beam is completely blocked within the respective mirror objective, is does not contribute to the microscope image. Therefore, the background is very dark or black. However, scattered light components that are bent or reflected by the specimen can pass the objective over its full aperture, and are not blocked by the light stop, because their optical pathway is different from the optical axis and the central illuminating beam (fig. 5 and 6).

In "axial dark field," the specimen is illuminated very homogenously. It appears like a self-luminous body, similar to fluorescence microscopy. Because of the extremely small aperture of the illuminating apparatus, the central illuminating light beam is much more coherent than in other modes, and the visible depth of field is dramatically enhanced. Although the condenser iris diaphragm is in maximum closed position, the lateral resolution is not visibly reduced, as the full aperture of the mirror lens remains effective for
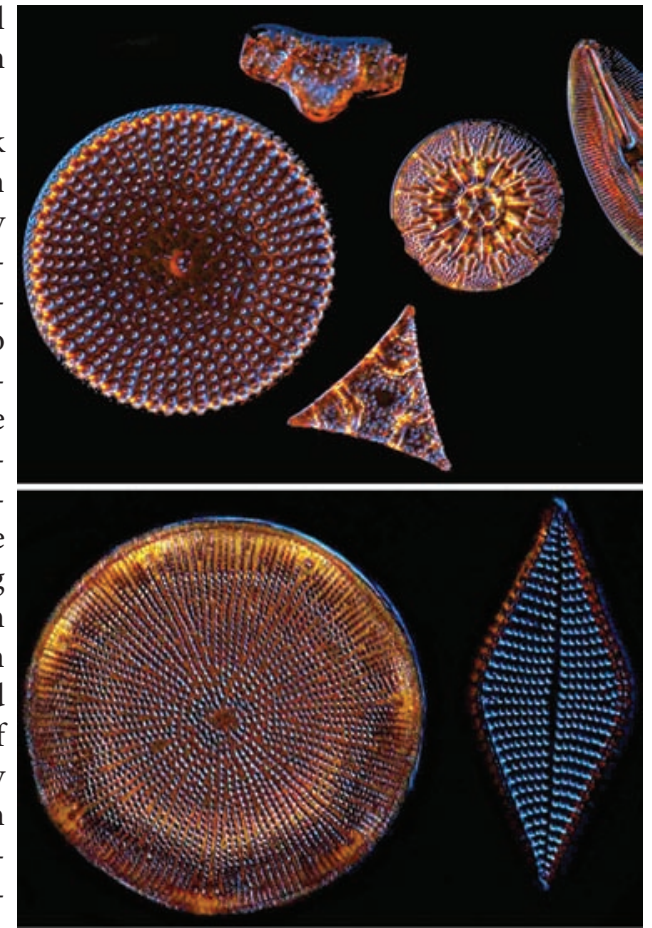

Top - Fig. 15: Diatoms, objective 20×, concentric aperture reduction darkfield in transmitted monochromatic blue light (H-beta filter, $\lambda=486 \mathrm{~nm}$ ), simultaneous darkfield in epiillumination (halogen bulb light), HFW: $0.30 \mathrm{~mm}$

Bottom - Fig. 16: Diatoms, objective 20x, illumination from fig. 15, HFW: $0.20 \mathrm{~mm}$ 
imaging. The numerical aperture is lower in mirror systems than in corresponding glass lenses. Nevertheless, sharpness and resolution remain excellent, because the illuminating light is more coherent and the specimen appears more luminescent. As the specimen is visible in high contrast with very homogeneous luminance, axial dark field illumination has also been called "luminance contrast." Some more particular optical and technical aspects of this illumination mode have already been published in prior papers $[8,9]$.
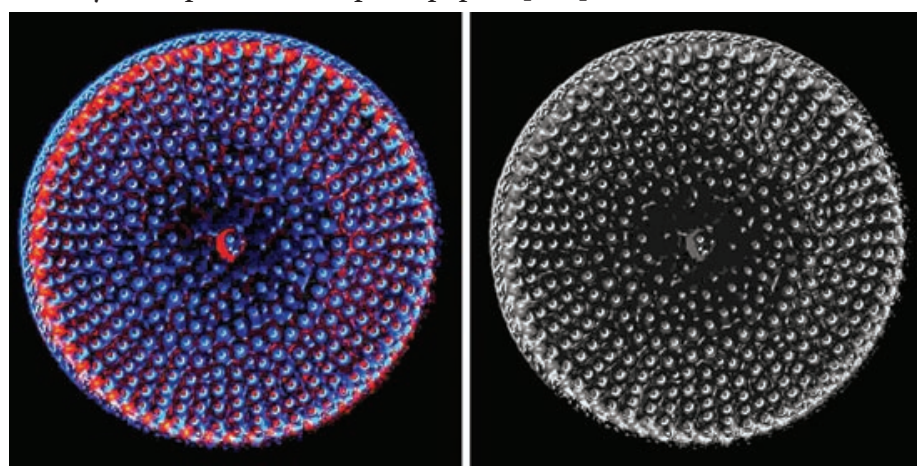

Fig. 17: Diatom, objective 20 $\times$, concentric aperture reduction darkfield in transmitted monochromatic blue light (H-beta filter, $\lambda=486 \mathrm{~nm}$ ), simultaneous epi-darkfield, filtered in red (H-alpha filter, $\lambda=656 \mathrm{~nm}$ ), HFW: $0.30 \mathrm{~mm}$, true color image (left) and B\&W image (right)

\section{Implementation of "digitized interference contrast"}

Interference contrast is a very suitable technique for three-dimensional imaging in small transparent specimens as fine structures usually appear in a distinctive three-dimensionality. Conversely, the depth of focus is minimized in interference contrast, as the vertical resolution is lower than in most other illumination modes. When three-dimensional specimens have to be presented in interference contrast over the full range of their vertical dimension, a sequence of several single images can be made, each taken in different focus. By this means, an image stack is created showing a vertical scan of the specimen. By use of suitable software, the single images can be superimposed with each other so that only zones that are in focus are selected for image processing. All pre-selected, in-focus parts, of the single images are finally assembled to a new deep focus image free of out-of-focus components. In this way, the three-dimensional aspect of transparent specimens can be fundamentally improved, especially in interference contrast images. The plasticity of specimens can often be accentuated when the background is turned into black by software-based postprocessing ("dark field interference contrast"). According to our own experiences in this, at least three software solutions are well suited for deep focus imaging in light microscopy: Combine Z 5 [4], Picolay [1] and Helicon Focus [5]. The mode of action and use of this software has already been described [7].

\section{Use of HDR techniques in dark field imaging}

Most darkfield images are characterized by a very high range of brightness and contrast, i.e. ultra high dynamic ranges. The dynamic range is defined as the ratio between the maximum and minimum values of luminance within an image. High dynamic ranges can be well perceived by the human eye, but they cannot be adequately presented by screens, printers, CCD chips or photographic films. Therefore, in conventional techniques (photographs, prints, monitor-based observations), dark zones within a specimen may be underexposed whereas bright parts may appear overexposed. Loss of visual information results from this. In photomicrography, the software Photomatix Pro can be used for high dynamic range rendering (HDR) promising best results with regard to the balance of brightness and contrast. When this rendering procedure is carried out, at least two single images have to be taken from the specimen with different exposures. These images are superimposed by Photomatix Pro, so that a high dynamic range image is reconstructed. While the usual digital 8-bit images are based on 256 tonal values, the number of tonal values in reconstructed HDR-images can be enlarged up to circa 4.3 billion graduations per channel. This high range of tonal values can neither be printed nor observed on normal screens. Because of this reason, the reconstructed HDR-image has to be transformed into a new image with a lower dynamic range. This procedure is called tone mapping. When tone mapping is carried out, the resulting final image shows a higher degree of sharpness, detail and fine structure over the full range of luminance, and it is free from any visible over- or underexposed zones. Fundamental improvements of the global quality can result from this technique, especially in darkfield images. Some more aspects about the handling of HDR techniques are published elsewhere $[10,11]$.

\section{Results}

By "condenser aperture reduction dark field," the visible depth of field can be significantly enhanced in life observations as well as in photomicrography when compared with traditional darkfield illumination. Marginal blooming that appears as an artifact in most darkfield images can be mitigated or completely eliminated by closing the condenser iris diaphragm. Peripheral scattered light components are reduced in the same way by closing the condenser diaphragm. Therefore, in most cases, an additional separate diaphragm mounted in an appropriate position (see plane $3^{\prime}$ in fig. 2 and plane 3 in fig. 3b) will not be needed. Overall, the condenser diaphragm does double duty as both an aperture and a field diaphragm. When the objective is fitted out with an integrated iris diaphragm, typically mounted in the back focal plane (see plane A in fig. 2 and plane 8 in fig. 3 ), the depth of field can be increased still more. The three-dimensional relief of some specimens can be visualized in an improved manner when "condenser aperture reduction darkfield" is carried out in the oblique illumination technique (eccentric variant). Fig. 7-13 demonstrate all relevant optical effects that are achievable by the various modifications of "condenser aperture reduction darkfield" described above.
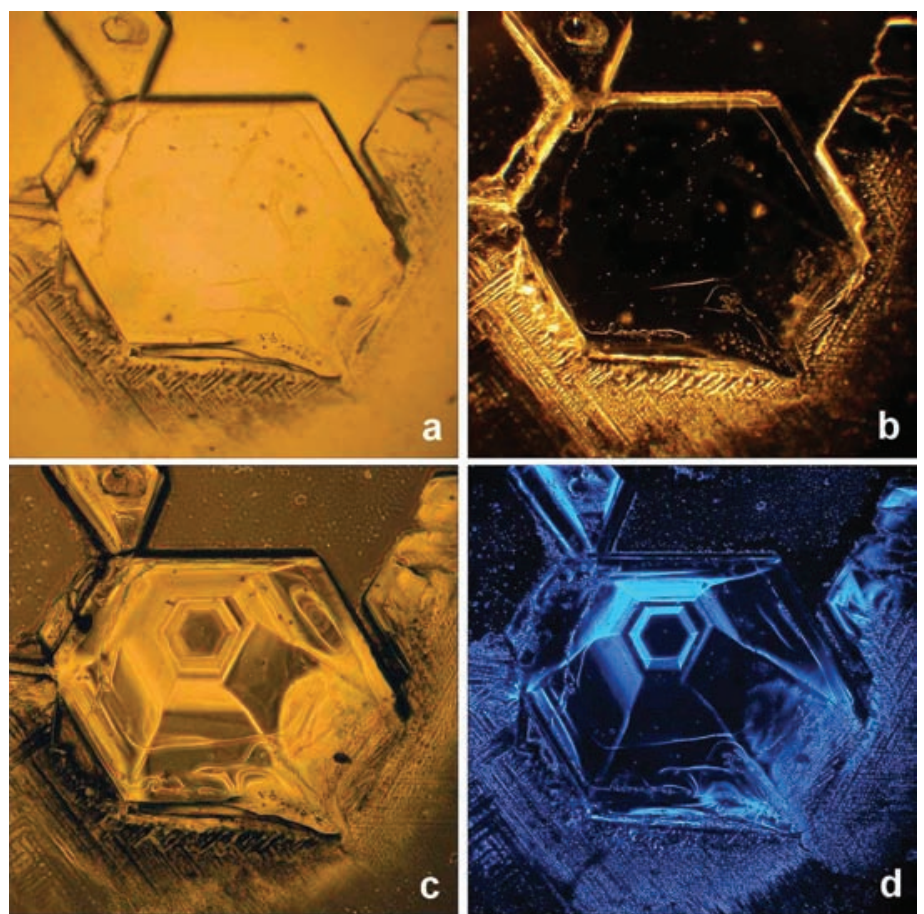

Fig. 18: Alum crystallization, preparation without coverslip, comparison of several illumination modes in transmitted light, planeapochromatic lenses 10×, NA 0.30 (images a-c), mirror objective 16x, NA 0.32 (image d). a: brightfield, b: conventional darkfield, c: phase contrast, $d$ : axial darkfield (luminance contrast), filtered in monochromatic blue light (H-beta filter, $\lambda=486 \mathrm{~nm}$ ). HFW: $0.70 \mathrm{~mm}$ 

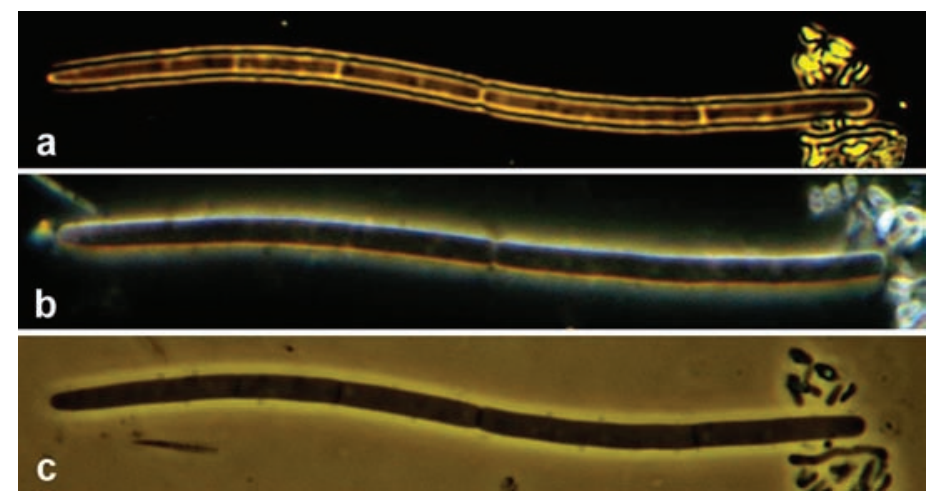

Fig. 19: Native dental bacillus, live preparation, comparison of several illumination modes in transmitted light, mirror water immersion $125 \times$, NA 0.90 (fig. a), plane-apochromatic oil immersions 100x, NA 1.32 (fig. $b$ and c). a: axial darkfield (luminance contrast), b: conventional darkfield, c: phase contrast. HFW: $0.06 \mathrm{~mm}$.

When darkfield imaging is obtained by epi-illumination, fine superficial structures in some transparent or semi-transparent specimens may also be visible in high clarity (fig. 14). Existing details in texture can be accentuated when darkfield in epi-illumination is combined with condenser aperture reduction darkfield illumination in transmitted light ("superimposed darkfield"). In this case, additional improving effects can be achieved when both illuminating light components are filtered in different colors, preferably by the use of monochromatic filters (fig. $15,16)$. Bicolor monochrome images created in superimposed darkfield imaging yields superior black and white images (fig. 17).

Axial darkfield in luminance contrast, carried out with mirror objectives, leads to the strongest enhancements of the visible depth of field. The character of deep focus imaging resulting from extremely small and coherent illuminating light beams might be reminiscent of confocal microscopy. When compared with other darkfield techniques, specimens are illuminated most homogeneously and are lit up like luminescent bodies. Because of these facts, fine detail inside a specimen can be visible in optimized clarity, sharpness, and resolution - nearly comparable with the optical effects in fluorescence microscopy. As mirror systems are characterized by a complete absence of chromatic and spherical aberration, optimized image planarity and sharpness, and a very high saturation and purity of colors result. Working distances are longer than in comparably glass lens microscopes. For example, the working distance of the COOKE mirror objective is $15 \mathrm{~mm}$, and the $125 \times$ magnifying water immersion works from a $0.5 \mathrm{~mm}$ distance.

Fig. 18 shows a quad screen view of a crystallization prepared without a coverslip, examined in transmitted light and epi-illumination. The pyramidal structure of this specimen is only visible in phase contrast and axial darkfield (luminance contrast), and luminance contrast leads to the highest grade in sharpness, resolution and visible depth of field. Fig. 19 demonstrates the superior quality of axial darkfield in ultra high magnification. A bacterial cell was taken in conventional darkfield and phase contrast carried out with $100 \times$ magnifying high-end plane-apochromatic glass lenses (oil immersions, numerical aperture: 1.32 ), and also in luminance contrast, using the $125 \times$ magnifying water immersion (mirror system, numerical aperture: 0.90). In luminance contrast (axial darkfield), most detail within the bacterium can be detected in best quality.

All advantages of axial darkfield are also relevant in polarization techniques. Fig. 20-22 show some views of ascorbic acid taken with the 50× magnifying COOKE mirror system from a $15 \mathrm{~mm}$ working distance. The extraordinary quality of this special construction is visible in normal halogen light illumination (fig. 20) as well as in polarized light (fig. 21, 22).
Digitized interference contrast can lead to final image reconstructions that show higher visual quality and information than in single shot images. Especially in transparent specimens with a large vertical dimension, their three-dimensional architecture can be imaged in an improved manner (fig. 23, 24).

Ultra-large local thickness and existing ultra-high ranges of brightness and contrast can both degrade visual information. Thus, in all techniques, the finally resulting image quality can be optimized furthermore by software-based stacking and HDR techniques if necessary (fig. 25).

\section{Discussion}

In several fields of light microscopy, the various techniques described in this article (concentric and eccentric condenser aperture reduction darkfield, superimposed and axial darkfield, digitized darkfield interference contrast) may promise improved quality in visual observations and photomicrographs when compared with conventional darkfield illumination and other concurrent examination methods including phase contrast und traditional interference contrast. Especially transparent or semi-transparent specimens with high ranges in local thickness, complex three-dimensional architecture and fine detail in their superficial structure may often be presented in a superior manner. Overall, a renaissance of dark field imaging could be forthcoming if these techniques were more widely used in the future.

To obtain condenser aperture reduction darkfield, manufacturers could create special condensers, modified in their optical design, so that the positions or projection planes of condenser diaphragm and field diaphragm are displaced as shown in fig. 2 and 3. A design drawing for a prototype of such a condenser specially constructed for aperture reduction darkfield is presented in fig 26 . The superior lens group (1) is designed as a Zoom system that can be manually regulated by a handle (2) so that the focal intercept can be continuously changed (arrow a). The condenser turret with a kit of several annular rings (3) is situated as usual in universal condensers. The aperture diaphragm (4) can be shifted in the vertical direction (arrow b), so that the best position for aperture reduction darkfield effects can be ascertained. A separate iris diaphragm (5) situated near the aperture diaphragm can act as additional
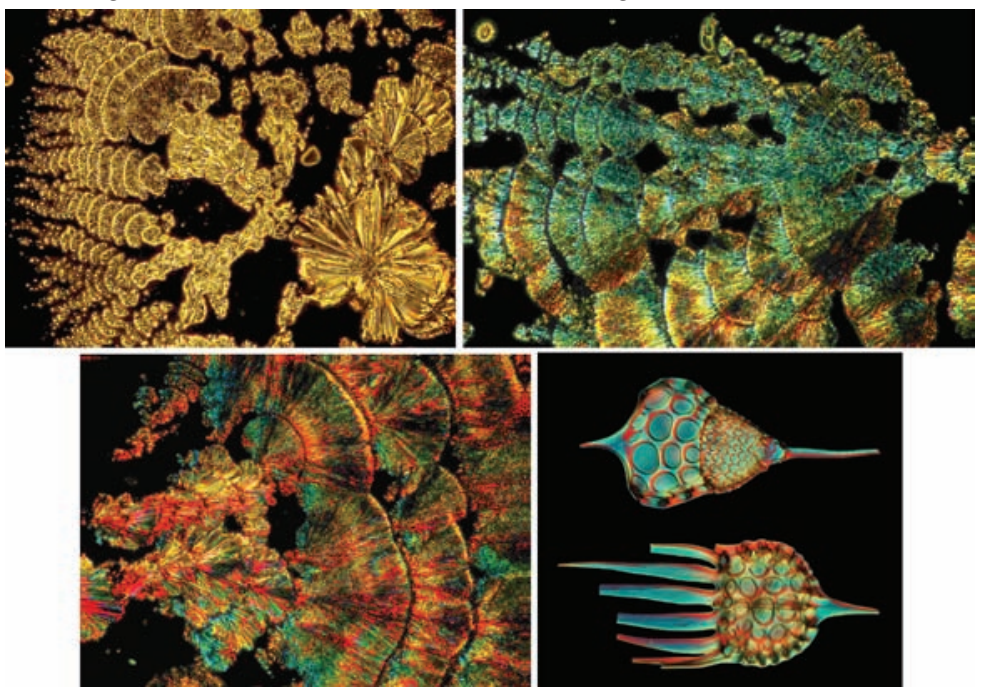

Fig. 20: Ascorbic acid, COOKE mirror objective 50×, working distance: 15 $\mathrm{mm}$, axial darkfield (luminance contrast), halogen bulb light. HFW: $0.2 \mathrm{~mm}$ (photograph taken by Timm Piper)

Fig. 21: Specimen and equipment from fig. 20, polarized light, polarizer and analyzer in crossed position. HFW: $0.2 \mathrm{~mm}$ (photograph taken by Timm Piper)

Fig. 22: Specimen and equipment from fig. 21, polarized light, lambda compensator. HFW: $0.2 \mathrm{~mm}$ (photograph taken by Timm Piper)

Fig. 23: Radiolarians, digitized interference contrast, objective 16x, superposition of 20 single images taken in different focus (Software: Combine Z 5), HFW: $0.30 \mathrm{~mm}$ 


\section{MIRA $\|_{\text {Schottky FE-SEM }}$}

\section{Value and Excellence in SEMs}

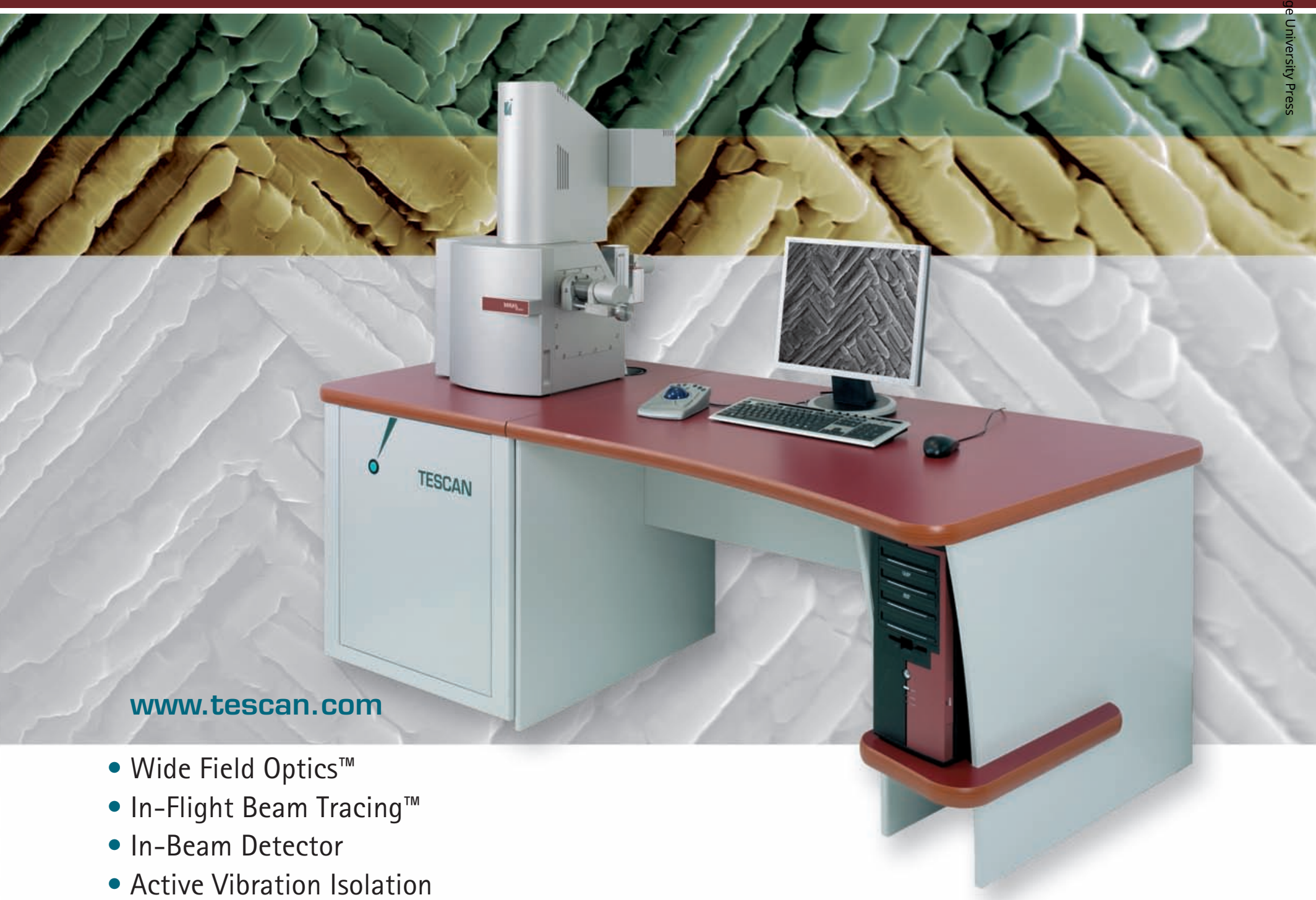

All as Tescan standard for the highest-quality imaging 
field diaphragm if desired.

Mirror systems might be of more particular importance and interest in the future because of their specific technical advantages (no chromatic and spherical aberration, long working distances, existing centric beam stops for axial darkfield in luminance contrast). Thus, in analogy to astronomy, mirror objectives might be able to achieve optimized image quality superior to conventional lens objectives, especially, when used for axial dark field or luminance contrast. In principle, the specimen is observed by a mirror systems in the same way as a celestial body is imaged via an astronomical telescope. It can be regarded as a generally accepted fact that astronomic mirror systems lead to better results than lens-based refractors; especially magnification, resolution, sharpness and purity of colors are improved. Thus, for example, the Hubble-telescope is constructed as a mirror system and not as a refractor.
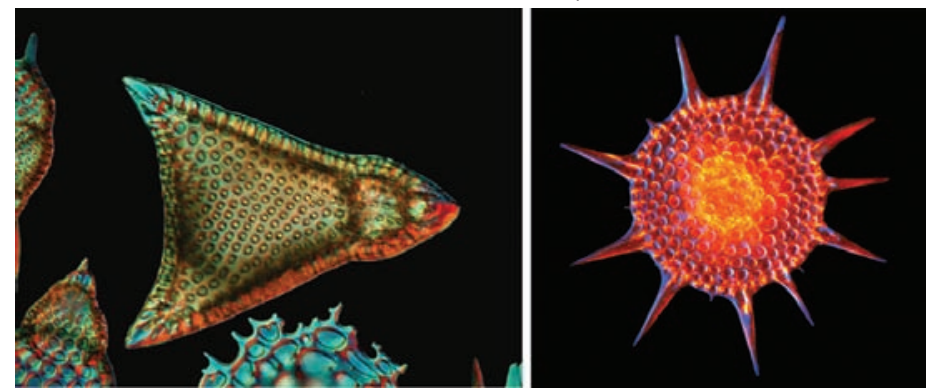

Left - Fig. 24: Radiolarians, digitized interference contrast, objective $16 \times$, superposition of 30 single images taken in different focus (Software: Combine Z 5), HFW: $0.40 \mathrm{~mm}$

Right - Fig. 25: Radiolarian, simultaneous aperture reduction darkfield illumination in transmitted light and epi-illumination, filtered in red and blue, HDR-reconstruction (postprocessing with Photomatix Pro), objective 20×, HFW: $0.15 \mathrm{~mm}$.

In microscopy, however, all lenses normally used are built up as refractors. On the other hand, the relationship between the sizes of microscopic specimens and the working distances of long distance microscope objectives can nearly be compared with astronomical dimensions. For example, with the help of a 40 or $50 \times$ COOKE mirror objective,

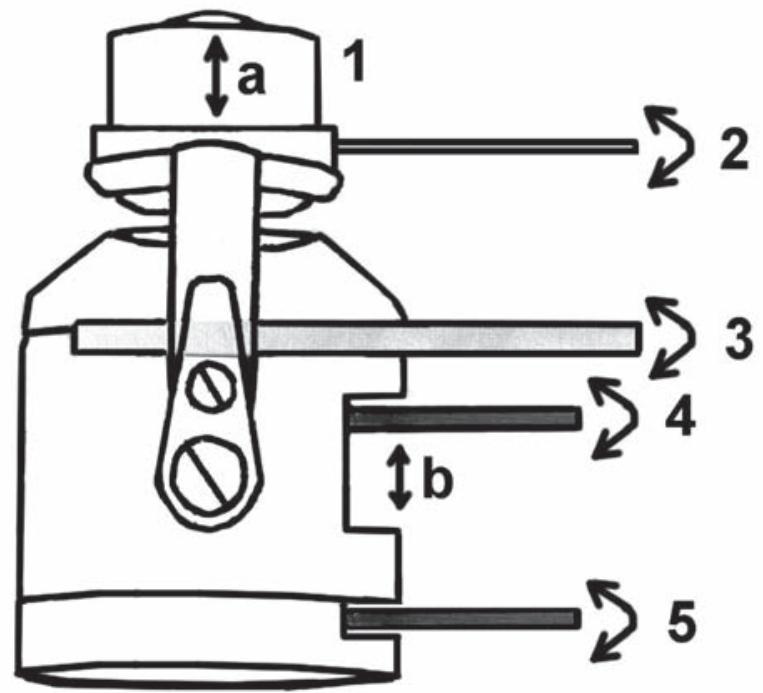

Fig. 26: Suggestion for a special condenser for aperture reduction darkfield (further explanations in the text)
$1=$ superior $z$ oom lens group
2 = handle for zoom setting
$3=$ turret with annular rings
$4=$ aperture diaphragm
$5=$ field diaphragm
arrow a: lens shift within the zoom system

arrow b: vertical shift of the aperture diaphragm specimens with a dimension of about 5 or $10 \mu \mathrm{m}$ can be observed well at a circa $15 \mathrm{~mm}$ working distance. In this case, the relation of the specimen size and the working distance is 1:1500 - 1:3000. Similar relations can also be calculated for modern stereomicroscopic glass lenses. The NIKON AZ 100 stereomicroscope, for instance, can be equipped with a $5 \times$ magnifying objective lens, NA 0.50 , working distance $15 \mathrm{~mm}$, so that a $500 \times$ total magnification is achievable (technical data published by the manufacturer).

The diameter of the moon is about $3500 \mathrm{~km}$, its distance from the earth about $350,000 \mathrm{~km}$. When the moon is observed from the earth, the relation of the object size and the observation distance is just about 1:100. The minimum distance from the planet Venus to the earth is about 40 million $\mathrm{km}$; the diameter of this planet is about $12,000 \mathrm{~km}$. For this celestial object, the relation of the object's size and distance is about 1 : 3000 when Venus is observed from the earth. These examples may demonstrate that the quantitative relations in microscopic examinations are similar to astronomical relations when high magnifying optical systems with very long working distances are used in microscopy.

A 40 or $50 \times$ COOKE mirror objective creates a 40 or 50 fold magnified primary image; when combined with a 10.0 or $12.5 \times$ magnifying eyepiece, a $500 \times$ total magnification will result. The modern high-end lens of the NIKON instrument, mentioned for example, is just able to create a 5 fold magnification of the primary image. Only with the help of additional tube lenses and eyepieces, a 500 $\times$ total magnification can be achieved. Moreover, the numerical aperture of the mirror objective is higher than in the stereoscopic lens objective ( 0.57 versus 0.50$)$. When the working distances and total magnifications are equal, a highly magnified primary image should lead to better results than a low magnified primary image, which needs a circa eight fold higher postmagnification to achieve an identically magnified secondary image.

It can be taken into account, as an additional argument for mirror objectives, that their light path remains constant for light components beyond the visible spectra because of their complete achromasia. Thus, axial dark field in luminance contrast could also be carried out when specimens are illuminated by extreme short wave blue or ultraviolet light. In this way, supramicroscopic resolution could be achieved using this technique.

\section{References:}

1. Cyprionka, H.: Picolay - freeware for generating improved images from picture series, 2007 http://www.picolay.de

2. Ernst Leitz Wetzlar GmbH: The imaging and illuminating optical elements in light microscopes - illuminators for epi-illumination (print in German), Wetzlar, 1969

3. HDR Soft: Photomatix, 2008 http://www.hdrsoft.com

4. Hedley, A.: CombineZ5. My software to combine pictures to increase depth of focus, 2007 http://www.hadleyweb.pwp.blueyonder.co.uk/index.htm

5. Heliconsoft: Helicon Focus, 2007 http://www.heliconfocus.com/heliconfocus. html

6. Linkenheld, Ch.: Theoretical basics of KÖHLER illumination (in German), 2003http://www.mikroskopie.de/kurse/koehlertheorie.htm

7. Piper, J.: Use of software to enhance depth of field and improve focus in photomicrographyMicroscopy and Analysis 22 (3), 15-19 (EU), 2008

8. Piper, J.: Luminance contrast - a new visible light technique for examining transparent specimens Microscopy Today, 15 (3), 26-34, 2007

9. Piper, J.: Luminance contrast, a new illumination technique in light microscopy: optical basics, practical evaluations, further developments, Opt. Int. J. Light Electron. Opt. doi: 10.1016/j.ijleo.2008.03.032, Elsevier, 2008

10. Piper, J.: Image processing for the optimization of dynamic range in photomicrography Microscopy and Analysis 23 (1), S9-S9 (EU), 2009.

11. Sasso, F.: How to create HDR-photos- HDR/Photomatix tutorial, 2007http:// abduzeedo.com/how-create-hdr-photos-hdrphotomatix-tutorial 


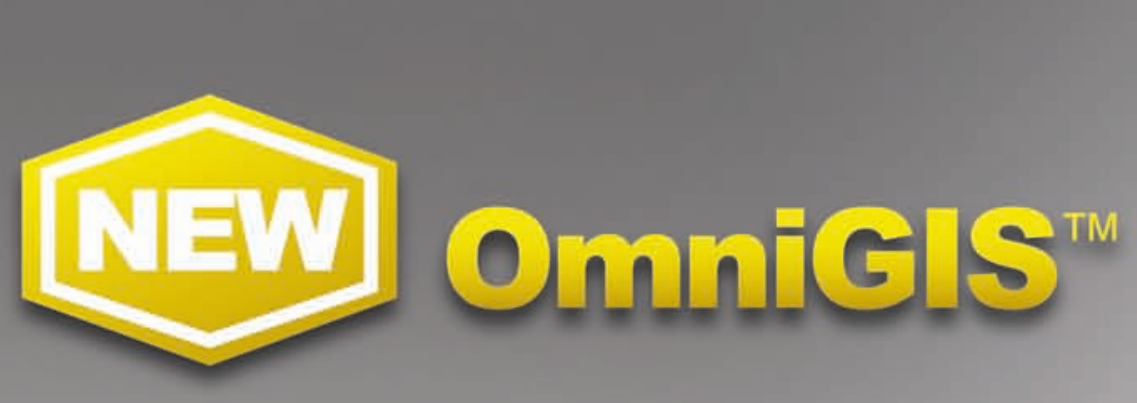

\section{Multi-Source}

Gas Injection System for the FIB \& SEM

FEATURES:

- Programmable Process Flows

- Feedback Control

- Single Needle Delivery

- Fast \& Easy Crucible Replacement

- Economical
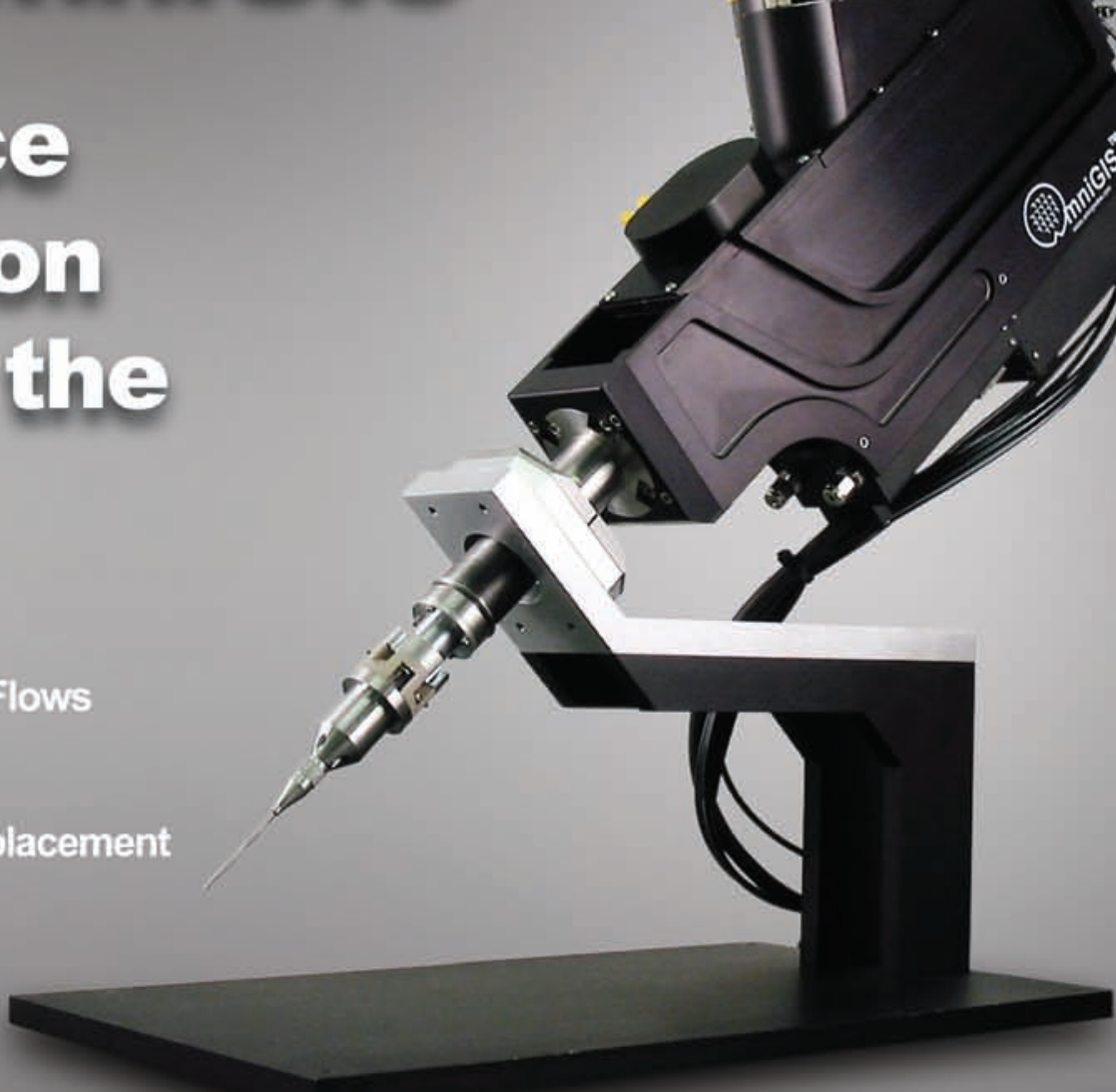

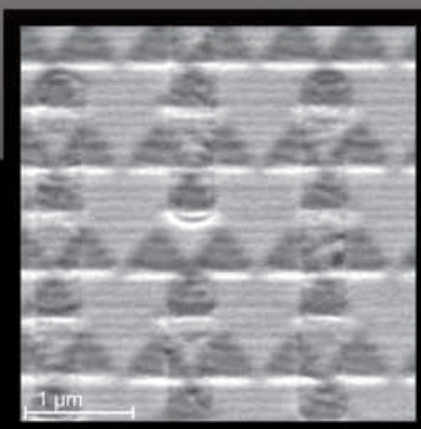

\section{Make}

FIB deposited plasmonic nanostructures

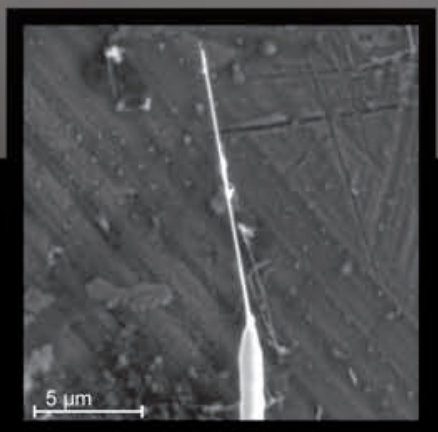

\section{Move}

Single nanowire analysis

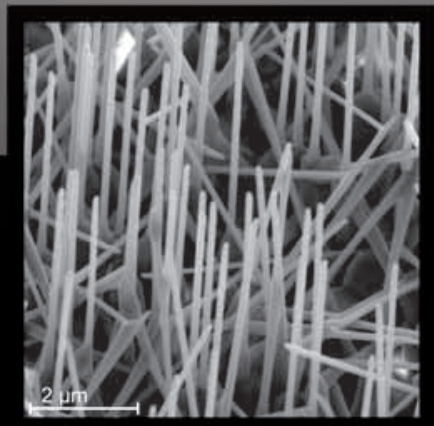

Modify

Chemical surface alteration

For more information visit us at http://www.omniprobe.com or call us at 214.570 .6800 .

\section{mniprobe}

www.omniprobe.com 